“ (C) 2017 IEEE. Personal use of this material is permitted. Permission from IEEE must be obtained for all other uses, in any current or future media, including

reprinting/republishing this material for advertising or promotional purposes, creating new collective works, for resale or redistribution to servers or lists, or reuse of any copyrighted component of this work in other works." 


\title{
A Novel Model Predictive Sliding Mode Control for AC/DC Converters with Output Voltage and Load Resistance Variations
}

\author{
Tingting He, Student Member, Li Li, Jianguo Zhu, Senior Member, IEEE and Linfeng Zheng
}

\begin{abstract}
This paper presents a novel model predictive sliding mode control (MPSMC) strategy for a three-phase grid connected AC/DC converter. The grid current is predicted for controlling the active and reactive power flows for the next sampling time instead of predicting them directly. This MPSMC scheme employs a sliding mode control (SMC) algorithm to calculate the reference values of active and reactive powers in the cost function. The reaching, existing and tracking conditions are analyzed to ensure that the designed sliding surface and control law are effective to control the system. The simulation results by Matlab/Simulink show that the MPSMC strategy is able to meet the system requirements of active and reactive powers, as well as the DC output voltage. Compared with the results obtained from the conventional model predictive PI control (MPPIC) scheme, the proposed strategy can improve the dynamic performance dramatically in terms of the response speed under system disturbances, such as varying output voltage and load demand.
\end{abstract}

Index Terms - Model predictive control; sliding mode control, PI control, AC/DC converter, system disturbances.

\section{INTRODUCTION}

Recently, a three-phase voltage source converter (VSC) has attracted significant attention due to its extensive applications and high-ranking position in the industrial power system [1]. It is available for bidirectional power flow, low harmonic line current, controllable power factor, and good DC-link voltage regulation ability. Therefore, it has been widely used in many areas, such as railway electrification systems [2], renewable energy systems (offshore wind power generation) [3], smart micro grids [4], and DC transmission systems [5]. In order to control these systems effectively, several control strategies have been studied and utilized, such as the voltage oriented control (VOC), the direct power control (DPC), and the model predictive control (MPC).

VOC is a well-known control strategy particularly appreciated for its excellent steady state performance and fast dynamic response. The classical VOC contains typically a double loop structure with the voltage control as the outer loop and current control as the inner loop. However, the dynamic performance of the converter depends strongly on the current loop and proportional-integral (PI) parameters $[1,6]$.

DPC employs a switching table to control the active and reactive powers simultaneously [7]. An optimal voltage vector is selected from this switching table [8]. Nonetheless, DPC always leads to large power ripples which reduce the power quality.

Currently, in terms of improving the system performance, the MPC strategy has been proposed for power converters [9]. MPC has several advantages, such as free of modulation, easy inclusion of system parameters, constraints and demands in the algorithm. Based on a system model, MPC selects an optimal voltage vector to predict the future state [10], and a PI controller is employed to generate the active power reference. Since the parameters of PI controller are fixed during the operation, the dynamic system performances are limited, especially under disturbances. When the system state varies, it is usually impossible to find a single set of PI control parameters of the model predictive PI control (MPPIC) method to meet all system requirements.

On the other hand, the sliding mode control (SMC) scheme exhibits excellent dynamic response. In addition, SMC is able to reject the uncertainties and disturbances, such as unknown variations of control variables and system parameters [11, 12]. It employs a control signal to guide the system trajectory points onto a surface, known as the sliding surface or hyper plane. All the trajectory points will be enforced to the vicinity of the sliding surface within a finite time and towards the system equilibrium point thereafter [13]. SMC is simple to implement and execute. A sliding surface and the control law are designed to achieve the desired objectives $[14,15]$.

This paper proposes a novel model predictive sliding mode control (MPSMC). SMC is introduced to compensate the drawbacks of the traditional PI controller used in MPC. Firstly, the system model of the AC/DC converter is investigated. Then, the theory of MPC strategy based on PI controller is described. The grid current is predicted to calculate the active and reactive powers for the next sampling time, which increases the calculation speed. A sliding surface and a control law are designed to control the $\mathrm{DC}$ voltage by regulating the active and reactive powers in an $\mathrm{AC} / \mathrm{DC}$ converter. Finally, the simulation results are carried out to observe and compare their dynamic performances obtained from the traditional and the proposed schemes.

\section{System Description AND Modeling}

Fig. 1 illustrates the power circuit of an AC/DC converter which is assumed as a three-phase voltage source rectifier. The main grid adopts the symmetrical three-phase three-wire system, connected with an IGBT full bridge through three 
series inductors $L$ and resistors $R$, connected with a capacitor $C$ to filter voltage harmonics on the DC side.

The active power conservation equation is used to describe the dynamic process of the voltage on the DC side, which can be calculated by

$$
P_{d c}=V_{d c} i_{d c}=C V_{d c} \frac{d}{d t} V_{d c}+\frac{1}{R_{L}} V_{d c}^{2},
$$

where $P_{d c}$ is the active power on DC side, and $V_{d c}$ and $i_{d c}$ are the converter output voltage and current, respectively.

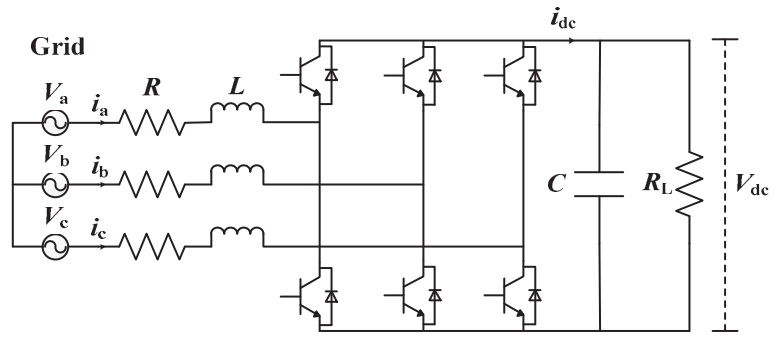

Fig. 1. AC/DC converter topology.

Based on the power equilibrium, the input active power of the converter is equal to its simultaneous output active power, which can be given by

$$
P_{a c}=P_{d c}+P_{\text {loss }},
$$

where $P_{\text {loss }}$ is the loss power of the converter, and in this paper, it is assumed to be zero.

It can be obtained from (1) and (2) that the active power on the $\mathrm{AC}$ side is equal to that on the $\mathrm{DC}$ side, shown as

$$
P_{a c}=P_{d c}=V_{d c} i_{d c}=C V_{d c} \frac{d}{d t} V_{d c}+\frac{1}{R_{L}} V_{d c}^{2} .
$$

\section{Model Predictive CONTROL}

Fig. 2 shows the basic principle of the traditional MPC with PI control method. According to the theory of space voltage vector pulse width modulation (SVPWM), the converter input voltage can be controlled by eight vectors in the two-phase stationary $\alpha-\beta$ coordinate system based on eight possible switching states, as the following

$$
V_{i}=\left\{\begin{array}{ll}
\frac{2}{3} V_{d c} e^{j(i-1) \frac{\pi}{3}} & (i=1, \cdots, 6) \\
0 & (i=0,7)
\end{array} .\right.
$$

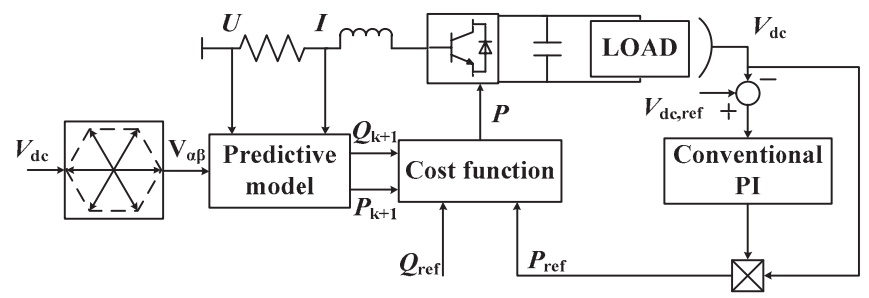

Fig. 2. Model predictive PI control scheme.

The mathematical model of the three-phase system in the two-phase stationary $\alpha-\beta$ coordinate system can be expressed as

$$
V_{g, \alpha \beta}=R i_{g, \alpha \beta}+L \frac{d i_{g, \alpha \beta}}{d t}+V_{i, \alpha \beta},
$$

where $V_{\mathrm{g}, \alpha \beta}$ and $i_{\mathrm{g}, \alpha \beta}$ are the grid voltage and current vectors expressed in stationary coordinates, respectively, and $V_{\mathrm{i}, \alpha \beta}$ is the converter input voltage.

The discrete-time model of the grid current at $(k+1)$ th instant for a sampling time $T_{\mathrm{s}}$ can be expressed as

$$
i_{g, \alpha \beta}(k+1)=\frac{T_{s}}{L}\left(V_{g, \alpha \beta}(k)-R i_{g, \alpha \beta}(k)-V_{i, \alpha \beta}(k)\right)+i_{g, \alpha \beta}(k) \text {. (6) }
$$

Then, the active and reactive powers of this system can be calculated by

$$
\left\{\begin{array}{l}
P=\frac{3}{2} \operatorname{Re}\left\{V_{g} i_{g}^{*}\right\}=\frac{3}{2}\left(V_{g, \alpha} i_{g, \alpha}+V_{g, \beta} i_{g, \beta}\right) \\
Q=\frac{3}{2} \operatorname{Im}\left\{V_{g} i_{g}^{*}\right\}=\frac{3}{2}\left(V_{g, \beta} i_{g, \alpha}-V_{g, \alpha} i_{g, \beta}\right)
\end{array} .\right.
$$

In order to control the active and reactive powers, the cost function $J$ can be designed as:

$$
J=\sqrt{\left(P-P_{r e f}\right)^{2}+\left(Q-Q_{r e f}\right)^{2}} .
$$

Substituting (4) and (6) into (7), one can deduce eight possible values for the active and reactive powers, separately, as the eight voltage vectors of the converter input voltage result in eight vectors of line current $i_{\mathrm{g}, \alpha \beta}$. The cost function is employed to select the optimal values of $P$ and $Q$, which can yield the minimum value of the cost function $J$. The corresponding voltage vector, that can generate the optimal values of $P$ and $Q$, is then selected for the next step switching.

Traditionally, the reference of active power $P_{\text {ref }}$ is determined by the DC output voltage utilizing the PI controller [7], and the reference of reactive power $Q_{\text {ref }}$ is set to be $0 \mathrm{VAr}$ to ensure the unity power factor operation. Therefore, according to (3), $V_{\mathrm{dc}}$ and $Q$ are the actual external control signals in controlling $P$ and $Q$. The main control block scheme is shown in Fig. 2.

\section{The Proposed CONTROL}

The proposed MPSMC strategy applies a sliding model controller to replace the PI controller, as shown in Fig. 3. The control problem is to detect an appropriate control law so that the system state can track the expected active power, as well as the desired dc-link voltage. In the steady state, the active power is proportional to the voltage on the DC side. Therefore, in order to control the active power, the objective of SMC can be converted to control the dc-link voltage.

\section{A. Active power sliding surface}

Based on the SMC theory, the sliding surfaces can be designed as a linear combination of the errors of state variables. Hence, the DC voltage tracking error is defined as the difference between the actual measured voltage $V_{d c}$ and the voltage reference $V_{d c, r e f}$,

$$
e_{V}=V_{d c}-V_{d c, r e f} .
$$

Then, the sliding surface is designed as the combination of the proportional and the integral of this voltage error, which is

$$
S_{V}=\lambda e_{V}+\int e_{V} d t
$$

where $\lambda$ is a positive constant. It is chosen to impose suitable switching state. 
Differentiating $S_{V}$ with respect to time and employ (10) to get

$$
\dot{S}_{V}=\lambda\left(\dot{V}_{d c}-\dot{V}_{d c, r e f}\right)+V_{d c}-V_{d c, r e f} .
$$

\section{B. Active power control law}

In this section, a MPSMC scheme for the AC/DC converter system is described and the control block diagram is depicted in Fig. 3. According to the inherent uncertainty with respect to the active power in this system, the control law can be defined as

$$
u= \begin{cases}P_{d c}^{+}(\mathrm{t}), & S_{V}>0, \\ P_{d c}^{-}(\mathrm{t}), & S_{V}<0 .\end{cases}
$$

where $u$ is the control law, $P_{d c}^{+}(t)$ and $P_{d c}^{-}(t)$ represent the instantaneous power when the sliding variable reach different sides of the sliding-mode surface $S_{V}$, respectively.

By defining the uncertainty disturbance $\delta$ in output voltage on DC side, the first-time derivation of voltage can be expressed as

$$
\dot{V}_{d c}=\frac{u}{C V_{d c}}-\frac{1}{R_{L} C} V_{d c}+\delta,
$$

where the bound of the uncertainty disturbance, $\rho$, is assumed to be $|\delta|<\rho<1$.

Based on the aforementioned analysis, the controller can be designed as

$$
u_{S_{V}}=C V_{d c}\left[\left(\frac{1}{R_{L} C}-\frac{1}{\lambda}\right) V_{d c}+\frac{1}{\lambda} V_{d c, r e f}-(\rho+k) \operatorname{sign}\left(S_{V}\right)\right],
$$

where $k>0$ represent the suitable control gain.

\section{Proof of Reaching Condition}

The reaching condition is to ensure that the system reaching point will reach the sliding surface in a finite time no matter where its initial position in the state space is $\left(S_{V}\right.$ would be negative, positive or zero) [16]. The control law is designed to meet the reach condition.

Apparently, if the converter output voltage is much higher than the reference voltage, i.e., $S_{\mathrm{V}}$ is positive, which means that the active power is less than the reference active power, and the switching state calculated by the cost function is controlled to increase the output voltage to the reference value so that more energy could be transferred from the AC source to the load. Conversely, if the converter output voltage is much lower than the reference voltage, i.e. $S_{\mathrm{V}}$ is negative, the switching state is then controlled to increase the output voltage to the reference value based on the proposed control law. The actual active power will be reduced to get the sliding surface in a finite time. Besides this, if the converter output voltage is equal to the reference value, i.e. $S_{\mathrm{V}}$ is zero, the switching state keeps constant to make sure the active power remains at the desired value. Therefore, the designed control law is efficient and can satisfy the reaching condition.

\section{Proof of Existence Condition}

The design of the sliding mode controller should also meet the existence condition. This guarantees that the state trajectory of reaching points in the neighborhood field of the switching surface is capable to reach the sliding surface within a finite period. It can be represented as

$$
\lim _{S_{V} \rightarrow 0} S_{V} \cdot \dot{S_{V}}<0 .
$$

Substituting (13) and (14) into (11), the sliding surface derivative can be rewritten as

$$
\dot{S_{V}}=-\lambda\left[(\rho+k) \operatorname{sign}\left(S_{V}\right)-\delta\right] .
$$

The sliding-mode existence condition can be verified as follows

$$
\begin{aligned}
S_{V} \dot{S}_{V} & =S_{V}\left(-\lambda\left((\rho+k) \operatorname{sign}\left(S_{V}\right)-\delta\right)\right) \\
& =-\lambda k\left|S_{V}\right|-\lambda S_{V}\left(\rho \operatorname{sign}\left(S_{V}\right)-\delta\right) .
\end{aligned}
$$

Therefore, the system is able to meet the existence condition via the following proof of two cases.

Case 1: if the reaching point is in a positive position in the neighborhood field of the created switching surface $S_{\mathrm{V}}$, i.e., $S_{\mathrm{V}}>0$,

$$
\begin{aligned}
S_{V} \dot{S}_{V} & =-\lambda k\left|S_{V}\right|-\lambda S_{V}\left(\rho \operatorname{sign}\left(S_{V}\right)-\delta\right) \\
& =-\lambda k\left|S_{V}\right|-\lambda S_{V}(\rho-\delta) \\
& <-\lambda k\left|S_{V}\right| \\
& <0 .
\end{aligned}
$$

This can lead to

$$
\lim _{S_{V} \rightarrow 0^{+}} S_{V} \dot{S_{V}}<0 .
$$

Case 2: if the reaching point is in a negative position in the neighborhood field of the created switching surface $S_{\mathrm{V}}$, i.e., $S_{\mathrm{V}}<0$,

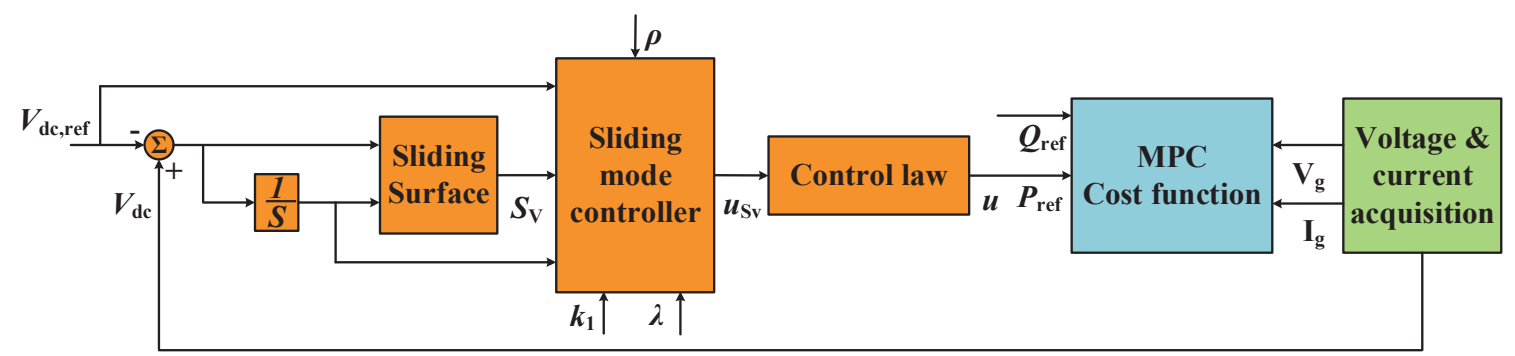

Fig. 3. Model predictive sliding mode control scheme. 


$$
\begin{aligned}
S_{V} \dot{S}_{V} & =-\lambda k\left|S_{V}\right|-\lambda S_{V}\left(\rho \operatorname{sign}\left(S_{V}\right)-\delta\right) \\
& =-\lambda k\left|S_{V}\right|+\lambda S_{V}(\rho+\delta) \\
& <-\lambda k\left|S_{V}\right| \\
& <0 .
\end{aligned}
$$

This results in

$$
\lim _{S_{V} \rightarrow 0^{-}} S_{V} \dot{S_{V}}<0 .
$$

Consider (19) and (21), it is easy to see that (15), the existence condition, can be satisfied as long as the sliding coefficients $\lambda$ and $k$ are efficiently selected.

\section{E. Proof of the tracking condition}

Substituting (14) into (13), when the system trajectory points reach the sliding surface, i.e., $S_{V}=0$, the uncertainty disturbance is equal to zero as well, i.e., $\delta=0$, then the first-time derivation of the voltage error can be rewritten as follows

$$
\dot{e}_{V}=\dot{V}_{d c}-\dot{V}_{d c, r e f}=-\frac{1}{\lambda}\left(V_{d c}-V_{d c, r e f}\right) .
$$

According to (22), as long as the sliding coefficient $\lambda$ is positive, then trajectory points can keep stable at the sliding surface theoretically. However, as the AC/DC converter is a nonlinear system, the positive $\lambda$ can hardly guarantee all the reaching points satisfy the stability condition globally, except for those points from the vicinity of the stability field. By applying the MPC and SMC schemes simultaneously in this system, all the trajectory points can hit and remain stable at the designed sliding surface only when $\lambda$ is chosen from an inherent range of the analyzed set, i.e., $\lambda>0$.

\section{Simulation Results}

\section{A. Simulation description}

The performance of the proposed MPSMC strategy is evaluated with a detailed AC/DC converter model carried out in Matlab/Simulink. The results are compared with the traditional MPPIC scheme in terms of tracking performances. The electric parameters of the AC/DC converter are reported in in Table I. Note that, when the desired DC voltage is $180 \mathrm{~V}$ and the resistance load is $150 \Omega$, it leads to an active power equal to 216 W. The reference of the reactive power $Q_{\text {ref }}$ is set to be $0 \mathrm{VAR}$ to ensure the unity power factor operation.

TABLE I

ELECTRIC PARAMETERS OF THE AC/DC CONVERTER

\begin{tabular}{llc}
\hline \hline Symbol & Quantity & Value \\
\hline$R$ & Grid line resistance & $0.25 \Omega$ \\
$L$ & Filter inductance & $10 \mathrm{mH}$ \\
$C$ & Filter capacitor & $470 \mathrm{uF}$ \\
$R_{L}$ & Load resistance & $150 \Omega$ \\
$V$ & Grid line-line voltage (RMS) & $100 \mathrm{~V}$ \\
$f$ & Gird frequency & $50 \mathrm{~Hz}$ \\
$V_{d c}$ & DC output voltage & $180 \mathrm{~V}$ \\
$T_{S}$ & Sample time & $50 \mu \mathrm{s}$ \\
\hline \hline
\end{tabular}

B. Steady and dynamic performance

Fig. 4 shows the behavior of the dc-link voltage of the $\mathrm{AC} / \mathrm{DC}$ converter when the output voltage requirement $180 \mathrm{~V}$ is applied on the DC side. It is possible to observe that the dynamic response of MPSMC is much more rapid than the conventional MPPIC strategy. The settling time (within $2 \%$ of the expected value) for MPSMC is about $0.015 \mathrm{~s}$, which is much shorter than $0.035 \mathrm{~s}$ of MPPIC method. The settling time is increased by $57 \%$ with the proposed MPSMC scheme. Moreover, the overshoot of the DC voltage by employing the MPSMC strategy can be limited and reduced dramatically, compared with the traditional MPPIC algorithm.

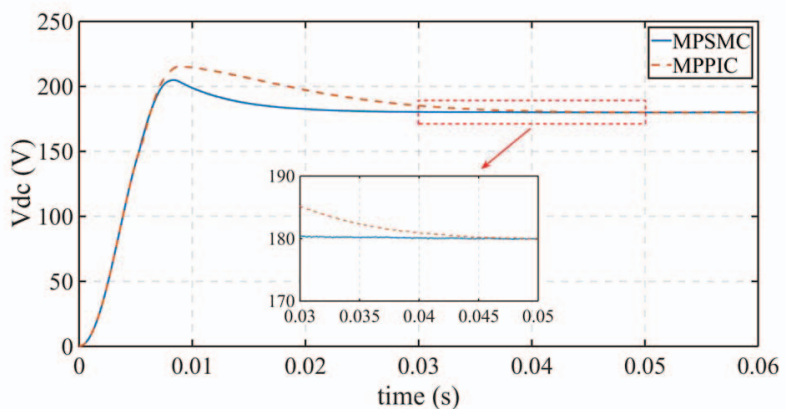

Fig. 4. DC voltage by applying MPPIC and MPSMC schemes.

Fig. 5 and 6 illustrate the simulated comparison of active and reactive powers using MPSMC and MPPIC strategies, respectively. It can be clearly seen that, for both methods, the active and reactive powers track the reference values very quickly, exhibiting very similar steady performance. However, the proposed MPSMC method brings more advantages in response speed, as shown in Fig. 6. The instantaneous AC currents on the grid side are shown in Fig. 7. The transient currents of MPSMC present the similar performance to MPPIC, with THD of $4.27 \%$ and $4.34 \%$, respectively.

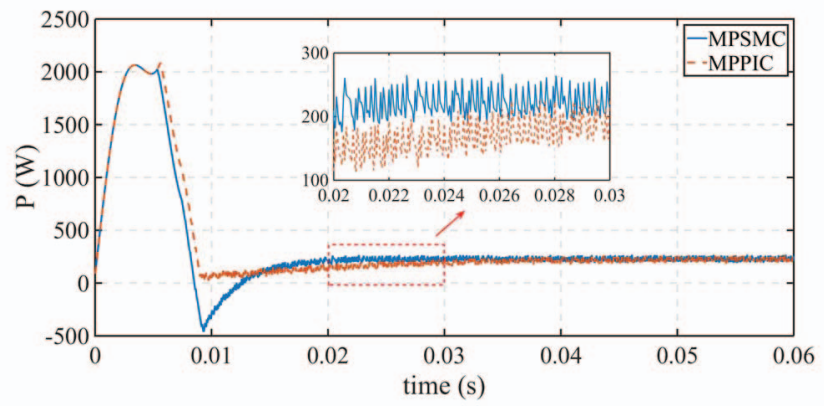

Fig. 5. Active power by applying MPPIC and MPSMC schemes.

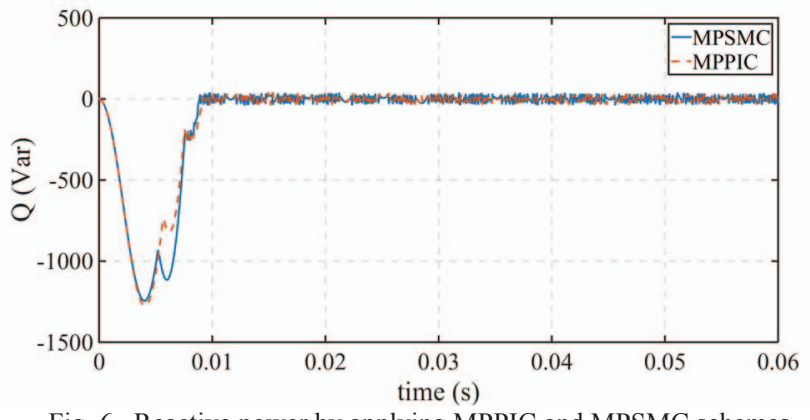

Fig. 6. Reactive power by applying MPPIC and MPSMC schemes. 

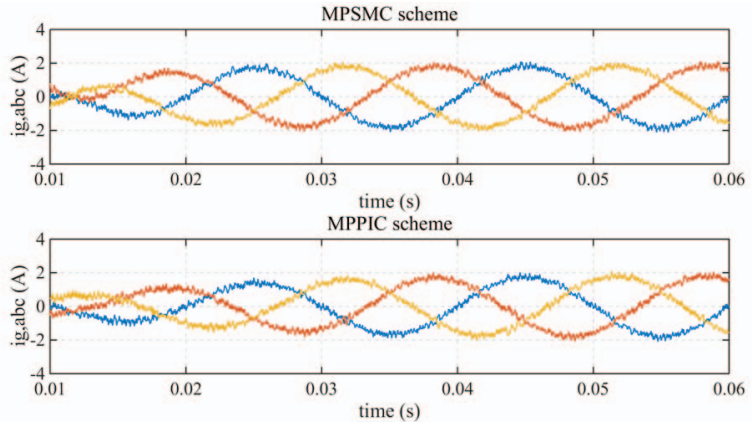

Fig. 7. Instantaneous AC currents by applying MPPIC and MPSMC schemes.

\section{Operation under demand variations of the load voltage}

Fig. 8 shows the behavior of the two methods used in the system when a negative load voltage demand step from $180 \mathrm{~V}$ to $150 \mathrm{~V}$ is applied at time instant $0.12 \mathrm{~s}$. It shows that the traditional MPPIC scheme takes $0.024 \mathrm{~s}$ to reach the steady state. The response time is nearly three times of that by using MPSMC (which is only $0.008 \mathrm{~s}$ ). Therefore, the proposed MPSMC method provides faster voltage tracking with much smaller overshoot to meet the updated requirement compared with the conventional controller.

Fig. 9 and 10 illustrate the performances of the active power, the reactive power and the grid currents under this demand variation, respectively. For the traditional MPPIC scheme, the active power spends more time getting a new steady state, equal to $150 \mathrm{~W}$.

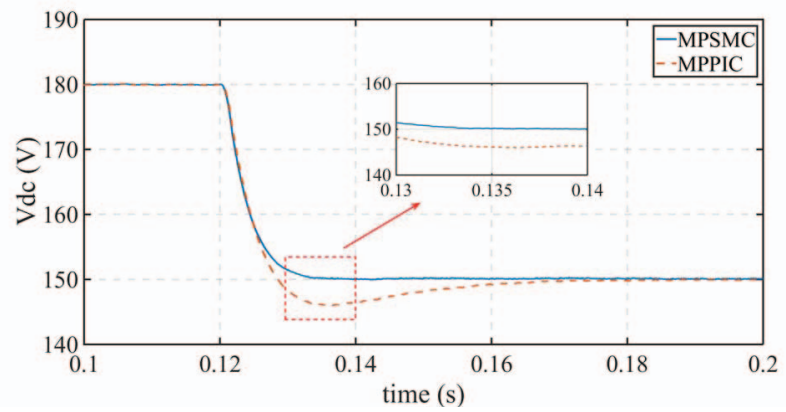

Fig. 8. Unexpected demand variation condition. DC voltage by applying MPPIC and MPSMC schemes.
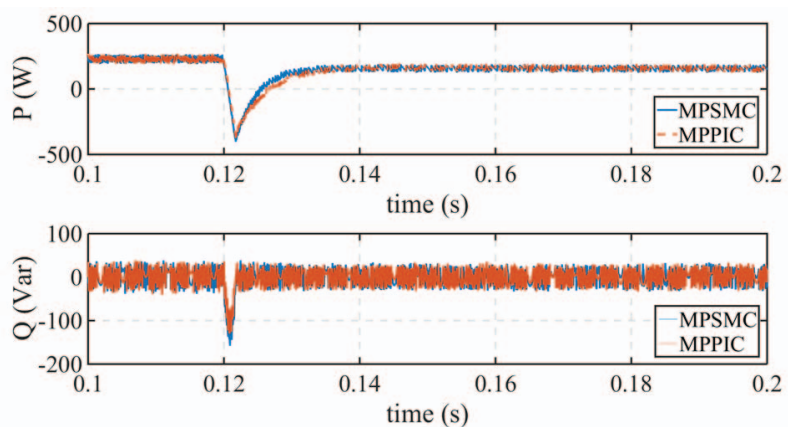

Fig. 9. Unexpected demand variation condition. Active and reactive powers by applying MPPIC and MPSMC schemes.

\section{Operation under variations of the load}

At time instant $0.32 \mathrm{~s}$, as shown in Fig. 11, a sharp decrement of the load changing from $300 \Omega$ to $150 \Omega$ is applied to the system. The MPPIC can track the original voltage reference with no error, but the response is relatively slow compared to the proposed MPSMC algorithm. The main advantage is that, because it is based on the system model, the MPSMC performance exhibits a fast response without any obvious variations on the load voltage, as well as the active power, shown in Fig. 12. Note that the transient currents on the grid side obtained from the MPSMC and MPPIC strategies present similar performance in Fig. 13.
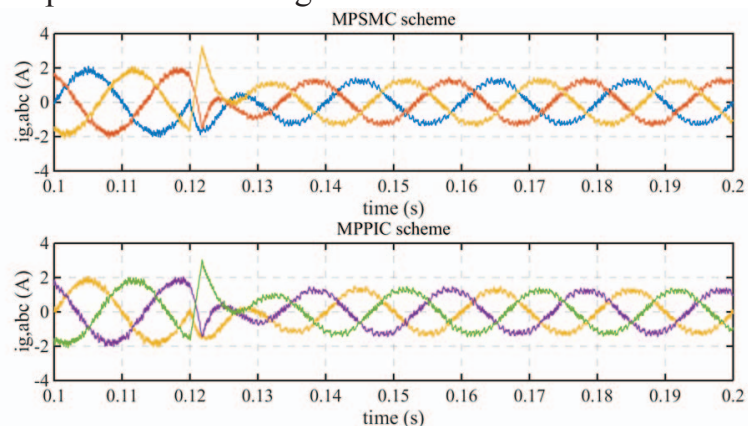

Fig. 10. Unexpected demand variation condition. Instantaneous AC currents by applying MPPIC and MPSMC schemes.

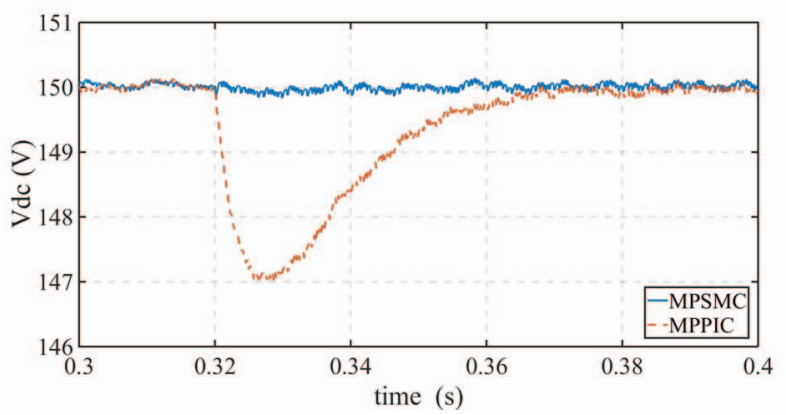

Fig. 11. Unexpected load variation condition. DC voltage by applying MPPIC and MPSMC schemes.
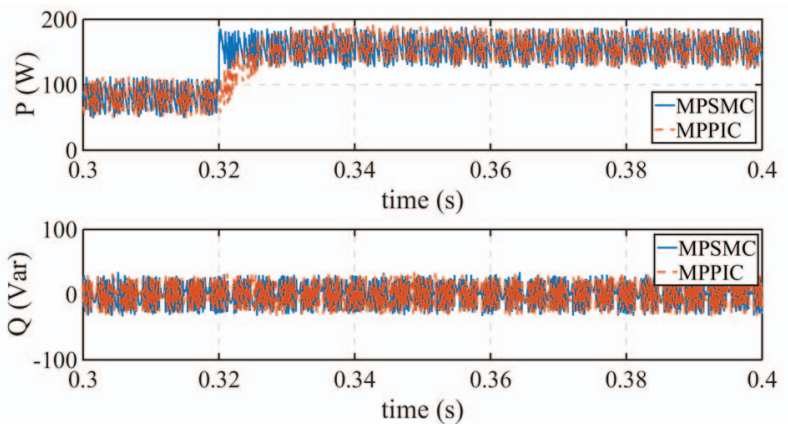

Fig. 12. Unexpected load variation condition. Active and reactive powers by applying MPPIC and MPSMC schemes.
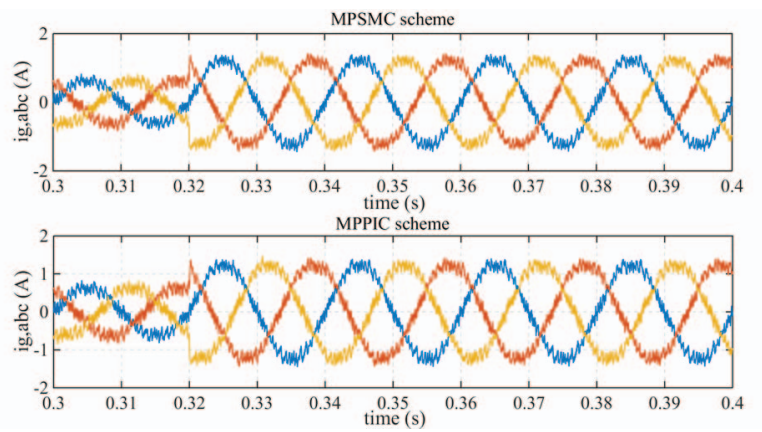

Fig. 13. Unexpected load variation condition. Instantaneous AC currents by applying MPPIC and MPSMC schemes. 


\section{CONCLUSION}

This paper presents a three-phase AC-DC converter controlled by the proposed model predictive sliding mode control versus the traditional MPPI control to generate the reference of active and reactive power. The simulation results show that while both two control strategies can meet the requirements of active and reactive powers, the proposed control scheme is able to increase the responding speed of establishing the stable voltage on the DC side, reduce the THD of the line current on the grid side, and respond more swiftly to the variation of the demand and the load. The proposed MPSMC only takes $15 \mathrm{~ms}$ to reach the stable level at the desired voltage, which is only half of the time by using the traditional MPPIC method. The THD can be reduced from $4.34 \%$ by PI control to $4.27 \%$ by SMC. Besides, great improvements are achieved when the demand and load fluctuate during the operation. This MPSMC scheme can also increase the efficiency for the main grid.

\section{REFERENCES}

[1] Y. Zhang, "Model predictive direct power control of a PWM rectifier with duty cycle optimization," IEEE Trans. Power Electron., vol. 28, no. 11, pp. 5343-5351, Nov. 2013.

[2] G. Antonio, "VSC-Based MVDC railway electrification system," IEEE Trans. Power Del., vol. 29, no. 1, pp. 422-431, Jan. 2014.

[3] H. Liu, "Contribution of VSC-HVDC to Frequency Regulation of Power Systems With Offshore Wind Generation," IEEE Trans. Energy Convers., vol. 30, no. 3, pp. 918-926, Sep. 2015.

[4] M. Davari, "Robust multi-objective control of VSC-based dc-voltage power port in hybrid ac/dc multi-terminal micro-grids," IEEE Trans. Smart Grid, vol. 4, no. 3, pp. 1597-1612, Mar. 2013.

[5] G. Venkataramanan, "A superconducting DC transmission system based on VSC transmission technologies," IEEE Trans. Appl. Supercond., vol. 13, no. 2, pp. 1922-1925, Feb. 2003.

[6] R. Kadri, "An improved maximum power point tracking for photovoltaic grid-connected inverter based on voltage-oriented control," IEEE Trans. Ind. Electron., vol. 58, no. 1, pp. 66-75, Jan. 2011.
[7] D. Choi, "Dynamic Performance Improvement of AC/DC Converter Using Model Predictive Direct Power Control With Finite Control Set," IEEE Trans. Ind. Electron., vol. 62, no. 2, pp. 757-767, Feb. 2015.

[8] Y. Zhang, "Performance Improvement of Two Vectors Based Model Predictive Control of PWM Rectifier," IEEE Trans. Power Electron., vol. 31, no. 8, pp. 6016-6030, Nov. 2016.

[9] C. Bordons, "Basic Principles of MPC for Power Converters: Bridging the Gap Between Theory and Practice," IEEE Industrial Electronics Magazine, vol. 9, no. 3, pp. 31-43, Sep. 2015.

[10] S. Kwak, "Model-Predictive Direct Power Control With Vector Preselection Technique for Highly Efficient Active Rectifiers," IEEE Transactions on Industrial Informatics, vol. 11, no. 1, pp. 44-52, Sep. 2015.

[11] J. Lee, "DSP-based sliding-mode control for electromagnetic levitation precise-position system." IEEE Transactions on Industrial Informatics, vol. 9, no. 2, pp. 817-827, Sep. 2015.

[12] S. Li, "Design and Implementation of Clutch Control for Automotive Transmissions Using Terminal Sliding-Mode Control and Uncertainty Observer," IEEE Transactions on Vehicular Technology, vol. 65, no. 4, pp. 1890-1898, April 2016.

[13] J. Zhang, "Adaptive sliding mode control for re-entry attitude of near space hypersonic vehicle based on backstepping design." IEEE/CAA Journal of Automatica Sinica, vol. 2, no.1, Jan. 2015.

[14] M. Cucuzzella, "Design of Robust Higher Order Sliding Mode Control for Microgrids," IEEE Journal on Emerging and Selected Topics in Circuits and Systems, vol. 5, no. 3, pp. 393-401, Sep. 2015.

[15] J. Jeronimo, "Development and implementation of a supervisor strategy and sliding mode control setup for fuel-cell-based hybrid generation systems," IEEE Transactions on Energy Conversion, vol. 30, no. 1, pp. 218-225, Mar. 2015.

[16] S. Tan, "General design issues of sliding-mode controllers in DC-DC converters," IEEE Trans. Ind. Electron., vol. 55, pp. 1160-1174, Mar. 2008. 